\title{
Common fixed point theorems for generalized contraction involving rational expressions in complex valued metric spaces
}

\author{
Hemant Kumar Nashine and Brian Fisher
}

\begin{abstract}
The purpose of this paper is to study common fixed points in complex valued metric spaces and obtain sufficient conditions for the existence of common fixed points of a pair of mappings satisfying generalized contraction involving rational expressions.
\end{abstract}

\section{Introduction}

The Banach contraction principle [4] is a very popular tool in solving existence problems in many branches of mathematical analysis. This famous theorem can be stated as follows.

Theorem 1.1. [4]. Let $(X, d)$ be a complete metric space and $T$ be a mapping of $X$ into itself satisfying:

$$
d(T x, T y) \leq k d(x, y), \forall x, y \in X,
$$

where $k$ is a constant in $(0,1)$. Then, $T$ has a unique fixed point $x^{*} \in X$.

Key Words: Common fixed point, contractive type mapping, complex valued metric space.

2010 Mathematics Subject Classification: Primary 54H25; Secondary 47H10.

Received: 20 June, 2013

Revised: 20 September, 2013

Accepted: 24 October, 2013 
There are in the literature a great number of generalizations of the Banach contraction principle (see $[1,2]$ and others). Some generalizations of the notion of a metric space have been proposed by some authors, such as, rectangular metric spaces, semi metric spaces, pseudo metric spaces, probabilistic metric spaces, fuzzy metric spaces, quasi metric spaces, quasi semi metric spaces, D-metric spaces, and cone metric spaces (see [5]- [10]).

Recently, Azam et al. [3] introduced the notion of complex valued metric spaces and established some fixed point results for mappings satisfying a rational inequality. In a continuation of Azam et al. [3], in this paper, we prove a common fixed point theorem for a pair of mappings satisfying a more general contraction involving rational expression in complex valued metric spaces.

\section{Preliminaries}

First of all, we introduce some notations and definitions that will be used later.

\subsection{Notations and Definitions}

The following definition was introduced by Azam et al. in [3].

Let $\mathbb{C}$ be the set of complex numbers and $z_{1}, z_{2} \in \mathbb{C}$. Define a partial order $\precsim$ on $\mathbb{C}$ as follows:

$$
z_{1} \precsim z_{2} \text { if and only if } \operatorname{Re}\left(z_{1}\right) \leq \operatorname{Re}\left(z_{2}\right), \operatorname{Im}\left(z_{1}\right) \leq \operatorname{Im}\left(z_{2}\right) \text {. }
$$

It follows that $z_{1} \precsim z_{2}$ if one of the following conditions is satisfied:

(i) $\operatorname{Re}\left(z_{1}\right)=\operatorname{Re}\left(z_{2}\right), \operatorname{Im}\left(z_{1}\right)<\operatorname{Im}\left(z_{2}\right)$,

(ii) $\operatorname{Re}\left(z_{1}\right)<\operatorname{Re}\left(z_{2}\right), \operatorname{Im}\left(z_{1}\right)=\operatorname{Im}\left(z_{2}\right)$,

(iii) $\operatorname{Re}\left(z_{1}\right)<\operatorname{Re}\left(z_{2}\right), \operatorname{Im}\left(z_{1}\right)<\operatorname{Im}\left(z_{2}\right)$,

(iv) $\operatorname{Re}\left(z_{1}\right)=\operatorname{Re}\left(z_{2}\right), \operatorname{Im}\left(z_{1}\right)=\operatorname{Im}\left(z_{2}\right)$.

In particular, we will write $z_{1} \lesssim z_{2}$ if $z_{1} \neq z_{2}$ and one of (i), (ii), and (iii) is satisfied and we will write $z_{1} \prec z_{2}$ if only (iii) is satisfied. Note that

$$
\begin{gathered}
0 \precsim z_{1} \lesssim z_{2} \Rightarrow\left|z_{1}\right|<\left|z_{2}\right|, \\
z_{1} \preceq z_{2}, z_{2} \prec z_{3} \Rightarrow z_{1} \prec z_{3} .
\end{gathered}
$$

Definition 2.1. Let $X$ be a nonempty set. Suppose that the mapping $d$ : $X \times X \rightarrow \mathbb{C}$, satisfies:

1. $0 \precsim d(x, y)$, for all $x, y \in X$ and $d(x, y)=0$ if and only if $x=y$;

2. $d(x, y)=d(y, x)$ for all $x, y \in X$;

3. $d(x, y) \precsim d(x, z)+d(z, y)$, for all $x, y, z \in X$.

Then $d$ is called a complex valued metric on $X$, and $(X, d)$ is called a complex valued metric space. 
A point $x \in X$ is called an interior point of a set $A \subseteq X$ whenever there exists $0 \prec r \in \mathbb{C}$ such that

$$
B(x, r)=\{y \in X: d(x, y) \prec r\} \subseteq A .
$$

A point $x \in X$ is called a limit point of $A$ whenever for every $0 \prec r \in \mathbb{C}$,

$$
B(x, r) \cap(A \backslash X) \neq \emptyset .
$$

$A$ is called open whenever each element of $A$ is an interior point of $A$. A subset $B \subseteq X$ is called closed whenever each limit point of B belongs to $B$. The family

$$
F=\{B(x, r): x \in X, 0 \prec r\} .
$$

is a sub-basis for a Hausdorff topology $\tau$ on $X$.

Let $\left\{x_{n}\right\}$ be a sequence in $X$ and $x \in X$. If for every $c \in \mathbb{C}$, with $0 \prec c$ there exists $n_{0} \in \mathbb{N}$ such that for all $n>n_{0}, d\left(x_{n}, x\right) \prec c$, then $\left\{x_{n}\right\}$ is said to be convergent, $\left\{x_{n}\right\}$ converges to $x$ and $x$ is the limit point of $\left\{x_{n}\right\}$. We denote this by $\lim _{n} x_{n}=x$, or $x_{n} \rightarrow x$, as $n \rightarrow \infty$. If for every $c \in \mathbb{C}$ with $0 \prec c$ there exists $n_{0} \in \mathbb{N}$ such that for all $n>n_{0}, d\left(x_{n}, x_{n+m}\right) \prec c$, then $\left\{x_{n}\right\}$ is called a Cauchy sequence in $(X, d)$. If every Cauchy sequence is convergent in $(X, d)$, then $(X, d)$ is called a complete complex valued metric space.

Lemma 2.2. [3]. Let $(X, d)$ be a complex valued metric space and let $\left\{x_{n}\right\}$ be a sequence in $X$. Then $\left\{x_{n}\right\}$ converges to $x$ if and only if $\left|d\left(x_{n}, x\right)\right| \rightarrow 0$ as $n \rightarrow \infty$

Lemma 2.3. [3]. Let $(X, d)$ be a complex valued metric space and let $\left\{x_{n}\right\}$ be a sequence in $X$. Then $\left\{x_{n}\right\}$ is a Cauchy sequence if and only if $\left|d\left(x_{n}, x_{n+m}\right)\right| \rightarrow$ 0 as $n \rightarrow \infty$.

\section{Common fixed point results in complete complex val- ued metric space}

Theorem 3.1. Let $(X, d)$ be a complete complex valued metric space and let the mappings $S, T: X \rightarrow X$ satisfy:

$$
\begin{aligned}
d(S x, T y) \precsim & \alpha d(x, y)+\frac{\beta[1+d(x, S x)] d(y, T y)}{1+d(x, y)} \\
& +\gamma[d(x, S x)+d(y, T y)]+\delta[d(x, T y)+d(y, S x)]
\end{aligned}
$$

for all $x, y \in X$, where $\alpha, \beta, \gamma, \delta$ are nonnegative reals with $\alpha+\beta+2 \gamma+2 \delta<1$. Then $S$ and $T$ have a unique common fixed point. 
COMMON FIXED POINT THEOREMS FOR GENERALIZED CONTRACTION INVOLVING RATIONAL EXPRESSIONS IN COMPLEX VALUED METRIC SPACES

Proof. Let $x_{0}$ be an arbitrary point in $X$ and define

$$
x_{2 k+1}=S x_{2 k}, \quad x_{2 k+2}=T x_{2 k+1}, k=0,1,2, \cdots .
$$

Then,

$$
\begin{aligned}
& d\left(x_{2 k+1}, x_{2 k+2}\right) \\
=\quad & d\left(S x_{2 k}, T x_{2 k+1}\right) \\
\precsim & \alpha d\left(x_{2 k}, x_{2 k+1}\right)+\frac{\beta\left[1+d\left(x_{2 k}, S x_{2 k}\right)\right] d\left(x_{2 k+1}, T x_{2 k+1}\right)}{1+d\left(x_{2 k}, x_{2 k+1}\right)} \\
& +\gamma\left[d\left(x_{2 k}, S x_{2 k}\right)+d\left(x_{2 k+1}, T x_{2 k+1}\right)\right]+\delta\left[d\left(x_{2 k}, T x_{2 k+1}\right)+d\left(x_{2 k+1}, S x_{2 k}\right)\right] \\
\precsim \quad & \alpha d\left(x_{2 k}, x_{2 k+1}\right)+\frac{\beta\left[1+d\left(x_{2 k}, x_{2 k+1}\right)\right] d\left(x_{2 k+1}, x_{2 k+2}\right)}{1+d\left(x_{2 k}, x_{2 k+1}\right)} \\
& +\gamma\left[d\left(x_{2 k}, x_{2 k+1}\right)+d\left(x_{2 k+1}, x_{2 k+2}\right)\right]+\delta\left[d\left(x_{2 k}, x_{2 k+2}\right)+d\left(x_{2 k+1}, x_{2 k+1}\right)\right] \\
\precsim \quad & \alpha d\left(x_{2 k}, x_{2 k+1}\right)+\beta d\left(x_{2 k+1}, x_{2 k+2}\right)+\gamma\left[d\left(x_{2 k}, x_{2 k+1}\right)+d\left(x_{2 k+1}, x_{2 k+2}\right)\right] \\
& +\delta\left[d\left(x_{2 k}, x_{2 k+1}\right)+d\left(x_{2 k+1}, x_{2 k+2}\right)\right] \\
\precsim \quad & \frac{\alpha+\gamma+\delta}{1-\beta-\gamma-\delta} d\left(x_{2 k}, x_{2 k+1}\right) .
\end{aligned}
$$

Similarly,

$$
\begin{aligned}
& d\left(x_{2 k+2}, x_{2 k+3}\right) \\
=\quad & d\left(S x_{2 k+1}, T x_{2 k+2}\right) \\
\precsim \quad & \alpha\left(x_{2 k+2}, x_{2 k+1}\right)+\frac{\beta\left[1+d\left(x_{2 k+1}, S x_{2 k+1}\right)\right] d\left(x_{2 k+2}, T x_{2 k+2}\right)}{1+d\left(x_{2 k+1}, x_{2 k+2}\right)} \\
& +\gamma\left[d\left(x_{2 k+1}, S x_{2 k+1}\right)+d\left(x_{2 k+2}, T x_{2 k+2}\right)\right] \\
& +\delta\left[d\left(x_{2 k+1}, T x_{2 k+2}\right)+d\left(x_{2 k+2}, S x_{2 k+1}\right)\right] \\
\precsim \quad & \alpha d\left(x_{2 k+1}, x_{2 k+2}\right)+\frac{\beta\left[1+d\left(x_{2 k}, x_{2 k+2}\right)\right] d\left(x_{2 k+2}, x_{2 k+3}\right)}{1+d\left(x_{2 k}, x_{2 k+2}\right)} \\
& +\gamma\left[d\left(x_{2 k+1}, x_{2 k+2}\right)+d\left(x_{2 k+2}, x_{2 k+3}\right)\right]+\delta\left[d\left(x_{2 k+1}, x_{2 k+3}\right)+d\left(x_{2 k+2}, x_{2 k+2}\right)\right] \\
& \precsim \quad \alpha d\left(x_{2 k+1}, x_{2 k+2}\right)+\beta d\left(x_{2 k+2}, x_{2 k+3}\right)+\gamma\left[d\left(x_{2 k+1}, x_{2 k+2}\right)+d\left(x_{2 k+2}, x_{2 k+3}\right)\right] \\
& +\delta\left[d\left(x_{2 k+1}, x_{2 k+2}\right)+d\left(x_{2 k+2}, x_{2 k+3}\right)\right] \\
\precsim \quad & \frac{\alpha+\gamma+\delta}{1-\beta-\gamma-\delta} d\left(x_{2 k+1}, x_{2 k+2}\right) .
\end{aligned}
$$

Putting

$$
h=\frac{\alpha+\gamma+\delta}{1-\beta-\gamma-\delta},
$$

we have

$$
d\left(x_{n+1}, x_{n+2}\right) \precsim h d\left(x_{n}, x_{n+1}\right) \precsim \cdots \precsim h^{n+1} d\left(x_{0}, x_{1}\right) .
$$


Hence, for any $m>n$,

$$
\begin{aligned}
d\left(x_{n}, x_{m}\right) & \precsim d\left(x_{n}, x_{n+1}\right)+d\left(x_{n+1}, x_{n+2}\right)+\cdots+d\left(x_{m-1}, x_{m}\right) \\
& \precsim\left[h^{n}+h^{n+1}+\cdots+h^{m-1}\right] d\left(x_{0}, x_{1}\right) \\
& \precsim \frac{h^{n}}{1-h} d\left(x_{0}, x_{1}\right)
\end{aligned}
$$

and so

$$
\left|d\left(x_{m}, x_{n}\right)\right| \leq \frac{h^{n}}{1-h}\left|d\left(x_{0}, x_{1}\right)\right| \rightarrow 0, \text { as } m, n \rightarrow \infty .
$$

This implies that $\left\{x_{n}\right\}$ is a Cauchy sequence. Since $X$ is complete, there exists $u \in X$ such that $x_{n} \rightarrow u$. It follows that $u=S u$, otherwise $d(u, S u)=z>0$ and we would then have

$$
\begin{aligned}
z & \precsim d\left(u, x_{2 k+2}\right)+d\left(x_{2 k+2}, S u\right) \\
& \precsim d\left(u, x_{2 k+2}\right)+d\left(T x_{2 k+1}, S u\right) \\
& \precsim d\left(u, x_{2 k+2}\right)+\alpha d\left(x_{2 k+1}, u\right)+\frac{\beta[1+d(u, S u)] d\left(x_{2 k+1}, T x_{2 k+1}\right)}{1+d\left(u, x_{2 k+1}\right)} \\
& \quad+\gamma\left[d(u, S u)+d\left(x_{2 k+1}, T x_{2 k+1}\right)\right] \\
& \quad+\delta\left[d\left(u, T x_{2 k+1}\right)+d\left(x_{2 k+1}, S u\right)\right] \\
& \precsim \quad d\left(u, x_{2 k+2}\right)+\alpha d\left(x_{2 k+1}, u\right)+\frac{\beta[1+d(u, S u)] d\left(x_{2 k+1}, x_{2 k+2}\right)}{1+d\left(u, x_{2 k+1}\right)} \\
& \quad+\gamma\left[d(u, S u)+d\left(x_{2 k+1}, x_{2 k+2}\right)\right]+\delta\left[d\left(u, x_{2 k+2}\right)+d\left(x_{2 k+1}, S u\right)\right] .
\end{aligned}
$$

This implies that

$$
\begin{aligned}
|z| \leq \quad & \left|d\left(u, x_{2 k+2}\right)\right|+\alpha\left|d\left(x_{2 k+1}, u\right)\right|+\frac{\beta|1+z|\left|d\left(x_{2 k+1}, x_{2 k+2}\right)\right|}{\left|1+d\left(u, x_{2 k+1}\right)\right|} \\
& +\gamma\left[|z|+\left|d\left(x_{2 k+1}, x_{2 k+2}\right)\right|\right]+\delta\left|d\left(u, x_{2 k+2}\right)+d\left(x_{2 k+1}, S u\right)\right| .
\end{aligned}
$$

Letting $n \rightarrow \infty$, it follows that

$$
|z| \leq(\gamma+\delta)|z| \leq(\alpha+\beta+2 \gamma+2 \delta)|z|<|z|,
$$

a contradiction and so $|z|=0$, that is, $u=S u$.

It follows similarly that $u=T u$.

We now show that $S$ and $T$ have unique common fixed point. For this, assume that $u^{*}$ in $X$ is a second common fixed point of $S$ and $T$. Then

$$
\begin{aligned}
d\left(u, u^{*}\right)= & d\left(S u, T u^{*}\right) \\
& \precsim \alpha d\left(u, u^{*}\right)+\frac{\beta[1+d(u, S u)] d\left(u^{*}, T u^{*}\right)}{1+d\left(u, u^{*}\right)}+\gamma\left[d(u, S u)+d\left(u^{*}, T u^{*}\right)\right] \\
& +\delta\left[d\left(u, T u^{*}\right)+d\left(u^{*}, S u\right)\right] \\
\precsim & (\alpha+2 \delta) d\left(u, u^{*}\right)
\end{aligned}
$$


and so $d\left(u, u^{*}\right)=0$, since $(\alpha+2 \delta)<1$. This implies that $u^{*}=u$, completing the proof of the theorem.

Putting $S=T$, we have

Corollary 3.2. Let $(X, d)$ be a complete complex valued metric space and let the mappings $T: X \rightarrow X$ satisfy:

$$
\begin{aligned}
d(T x, T y) \precsim & \alpha d(x, y)+\frac{\beta[1+d(x, T x)] d(y, T y)}{1+d(x, y)}+\gamma[d(x, T x)+d(y, T y)] \\
& +\delta[d(x, T y)+d(y, S x)]
\end{aligned}
$$

for all $x, y \in X$, where $\alpha, \beta, \gamma, \delta$ are nonnegative reals with $\alpha+\beta+2 \gamma+2 \delta<1$. Then $T$ has a unique fixed point.

Corollary 3.3. Let $(X, d)$ be a complete complex valued metric space and let the mappings $T: X \rightarrow X$ satisfy:

$$
\begin{aligned}
d\left(T^{n} x, T^{n} y\right) \precsim & \alpha d(x, y)+\frac{\beta\left[1+d\left(x, T^{n} x\right)\right] d\left(y, T^{n} y\right)}{1+d(x, y)}+\gamma\left[d\left(x, T^{n} x\right)+d\left(y, T^{n} y\right)\right] \\
& +\delta\left[d\left(x, T^{n} y\right)+d\left(y, T^{n} x\right)\right]
\end{aligned}
$$

for all $x, y \in X$, where $\alpha, \beta, \gamma, \delta$ are nonnegative reals with $\alpha+\beta+2 \gamma+2 \delta<1$. Then $T$ has a unique fixed point.

Proof. By Corollary 3.2 there exists $v \in X$ such that $T^{n} v=v$. Then

$$
\begin{aligned}
d(T v, v)= & d\left(T T^{n} v, T^{n} v\right)=d\left(T^{n} T v, T^{n} v\right) \\
\precsim & \alpha d(T v, v)+\frac{\beta\left[1+d\left(T v, T^{n} T v\right)\right] d\left(v, T^{n} v\right)}{1+d(T v, v)} \\
& +\gamma\left[d\left(T v, T^{n} T v\right)+d\left(v, T^{n} v\right)\right]+\delta\left[d\left(T v, T^{n} v\right)+d\left(v, T^{n} T v\right)\right] \\
\precsim & \alpha d(T v, v)+\frac{\beta\left[1+d\left(T v, T T^{n} v\right)\right] d(v, v)}{1+d(T v, v)} \\
& +\gamma d\left(T v, T T^{n} v\right)+\delta\left[d(T v, v)+d\left(v, T T^{n} v\right)\right] \\
= & (\alpha+2 \delta) d(T v, v)
\end{aligned}
$$

and so $d(T v, v)=0$.

\section{References}

[1] R.P. Agarwal, D. O'Regan, D.R. Sahu, Fixed point theory for Lipschitzian-type mappings with applications, Series: Topological Fixed Point Theorems and its Applications, 6, Springer, New York, 2009. 
COMMON FIXED POINT THEOREMS FOR GENERALIZED CONTRACTION INVOLVING RATIONAL

[2] M. A. Alghamdi, V. Berinde, N. Shahzad, Fixed points of non-self almost contractions, Carpathian Journal of Mathematics, 30(1)(2014), 7-14.

[3] A. Azam, B. Fisher, M. Khan, Common fixed point theorems in complex valued metric spaces, Numerical Functional Analysis and Optimization, 3(3) (2011), 243-253.

[4] S. Banach, Sur les opérations dans les ensembles abstraits et leur application aux équations intégrales, Fund. Math., 3(1922), 133-181.

[5] O. Hadzic, E. Pap, Fixed Point Theory in PM-Spaces, Kluwer Academic, Dordrecht, The Netherlands(2001).

[6] L.G. Huang, X. Zhang, Cone metric spaces and fixed point theorems of contractive mappings, J. Math. Anal. Appl., 332 (2007), 1468-1476.

[7] J.L. Kelley, General Topology, Van Nostrand Reinhold, New York(1955).

[8] H.P.A. Kunzi, A note on sequentially compact quasi-pseudo-metric spaces, Monatshefte Math., 95(1983), 219-220.

[9] M. Telci, B. Fisher, On a fixed point theorem for fuzzy mappings in quasimetric spaces, Thai J. Math., 2(2003), 1-8.

[10] J.S. Ume, Fixed point theorems in generalizing spaces of quasi metric family and applications, Indian J. Pure App. Math., 33(2002), 1041-1051.

Hemant Kumar NASHINE,

Department of Mathematics,

Disha Institute of Management and Technology,

Satya Vihar, Vidhansabha-Chandrakhuri Marg,

Mandir Hasaud, Raipur-492101(Chhattisgarh), India.

Email:drhknashine@gmail.com

Brian FISHER,

Department of Mathematics,

University of Leicester,

Leicester, England.

Email: fbr@leicester.ac.uk 